\title{
Avaliação da contribuição dos diferentes componentes da radiação cósmica atmosférica na dose em tripulações de aeronaves
}

\author{
M. A. Pereiraa, ${ }^{\mathrm{a}, \mathrm{b}}$ A. C. M. Prado ${ }^{\mathrm{a}, \mathrm{b}}$; C. A. Federico ${ }^{\mathrm{a}, \mathrm{b}}$; O. L. Gonçalez ${ }^{\mathrm{a}, \mathrm{b}}$ \\ a Instituto Tecnológico de Aeronáutica, 12228-900, São José dos Campos-São Paulo, Brasil \\ ${ }^{b}$ Divisão de Física Aplicada, Instituto de Estudos Avançados, 12228-001, São José dos Campos-São Paulo, Brasil \\ marlon@ieav.cta.br
}

\begin{abstract}
RESUMO
As tripulações e passageiros de aeronaves são expostos à radiação cósmica atmosférica. O fluxo destas radiações é modulado pelo ciclo solar e clima espacial, variando com a latitude geomagnética e com a altitude. Este trabalho apresenta um estudo das contribuições das radiações no equivalente de dose ambiente total de tripulações em função da altitude de voo até $20 \mathrm{~km}$, durante máximo e mínimo solar e em regiões equatorial e polar. Foram utilizados os resultados de cálculos dos fluxos de partículas gerados por meio dos códigos EXPACS e QARM. As partículas avaliadas que contribuem significativamente no equivalente de dose ambiente são os nêutrons, prótons, elétrons, pósitrons, alfas, fótons, múons e píons carregados. Esta avaliação permite caracterizar a origem da dose recebida por tripulações e, também, fundamentar um projeto de um sistema dosimétrico adequado para este campo de radiação ionizante no interior de aeronaves e em solo.
\end{abstract}

Palavras-chave: radiação cósmica, aeronautas, monitoramento da radiação.

\section{INTRODUCÃO}

Nas últimas décadas, o controle do nível de dose de radiação ionizante no ambiente aeronáutico e seus efeitos em tripulações e sistemas aviônicos tem se tornado muito mais significativo, motivando inúmeros estudos em diferentes áreas como saúde ocupacional, proteção radiológica, segurança de voo, entre outras (FEDERICO, 2011; FEDERICO, 2012; FEDERICO, 2012; 
FEDERICO, 2010; BARTLETT, 2004; HAJEK, 2004; LIM, 2002), evidenciando que os profissionais de aviação estão expostos a níveis de radiação da mesma ordem de grandeza a que os trabalhadores com radiação em medicina e áreas nucleares estão expostos (FEDERICO, 2010).

As taxas dose provenientes da radiação cósmica [RC] na atmosfera aumentam consideravelmente com a altitude podendo exceder o limite de dose anual para indivíduos do público proposto por organismos internacionais (FEDERICO, 2012).

A Comissão Internacional de Proteção Radiológica (ICRP) reconhece a necessidade de controle da exposição dos profissionais de voo, como pilotos e tripulação, considerando tais exposições comparáveis àquelas dos trabalhadores da área nuclear (INTERNATIONAL COMMISSION ON RADIOLOGICAL PROTECTION, 2007).

No Brasil, este assunto começou a ser abordado em torno de 2008 quando o Instituto de Estudos Avançados, pertencente ao Departamento de Ciência e Tecnologia Aeroespacial (IEAv/DCTA) do Comando da Aeronáutica, juntamente com a Comissão Nacional de Energia Nuclear, por meio de seus institutos de pesquisa, Instituto de Pesquisas Energéticas e Nucleares (IPEN) e o Instituto de Radioproteção e Dosimetria (IRD) (FEDERICO, 2010; FEDERICO, 2011; FEDERICO, 2012; FEDERICO, 2012b), iniciaram os primeiros estudos e medidas a bordo de aeronaves.

A radiação cósmica ( $\mathrm{RC}$ ) que atinge a terra possui uma componente galáctica (RCG) e uma componente proveniente do Sol (RCS). A componente galáctica possui, em ambiente solar, uma composição em torno de $98 \%$ de núcleos atômicos totalmente ionizados ( $88 \%$ prótons, $11 \%$ alfas e em torno de $1 \%$ de núcleos dos demais elementos presentes na natureza) e $2 \%$ de elétrons, enquanto que a componente solar (RCS), por sua vez, é composta basicamente de partículas provenientes de eventos solares ou SPE (solar particle events) (FEDERICO, 2010, HAJEK, 2004; DURANTE, 2011), consistindo principalmente de prótons e elétrons.

Além do campo magnético terrestre que atua como um defletor da RC incidente na terra e da atmosfera terrestre que atua como um atenuador desta radiação à medida que esta penetra as camadas mais densas da atmosfera, outros fatores também interferem na intensidade das componentes principais da radiação cósmica, a RCS e a RCG (FEDERICO, 2011), conforme apresentado com mais detalhes a seguir. 
A RCS provoca o espalhamento e a desaceleração da RCG de menor energia, de maneira que a intensidade relativa da RCS e RCG estão anticorrelacionas, ou seja: quando o Sol está em atividade máxima, a intensidade da RCG é mínima e vice-versa. Esta propriedade é conhecida potencial de desaceleração solar ou ainda, potencial heliocêntrico (medido em MV). O campo magnético da Terra, por sua vez, deflete parte das partículas de RC incidentes e, esta deflexão é maior na região do equador e menor nos polos, permitindo níveis de dose de duas a três vezes maiores que nas regiões equatoriais. Esta capacidade de deflexão das partículas em sua componente vertical é denominada de rigidez de corte vertical (FEDERICO, 2011).

As perturbações do campo magnético são indicadas de diversas formas, mas um dos índices mais utilizados para indicar a variação na componente magnética horizontal do campo geomagnético em um intervalo de três horas consecutivas é o índice $\mathrm{K}$, medido em 13 observatórios magnéticos distribuídos entre América do Norte, Europa e Oceania. O valor médio destes índices $\mathrm{K}$ é conhecido como índice global $\mathrm{Kp}$, que é muito utilizado em estudos ionosféricos e magnetosféricos e também por programas de cálculo de dose que consideram a variação da taxa de dose em decorrência de perturbações geomagnéticas.

As partículas da RC que penetram na atmosfera interagem com os núcleos dos componentes da atmosfera, produzindo uma cascata de radiações secundárias, por meio de reações nucleares de fragmentação destes núcleos (spallation). Uma única partícula primária de grande energia forma um verdadeiro "chuveiro" de radiação secundária que se expande por uma grande extensão (cerca $2 \mathrm{~km}$ de diâmetro na superfície do solo).

A contribuição dos diferentes tipos de partículas deste chuveiro no equivalente de dose ambiente varia em função da altitude. Na altitude de interesse para aviação, ocorre na seguinte ordem de importância: nêutrons, elétrons, prótons, múons e píons carregados (FEDERICO, 2011).

A grandeza operacional de proteção radiológica indicada para o monitoramento de área para radiações penetrantes é o equivalente de dose ambiente, $\mathrm{H}^{*}(10)$.

Há diversos códigos computacionais em uso atualmente para a estimativa da dose proveniente da radiação cósmica (FEDERICO, 2011). Neste trabalho foram utilizados dois códigos computacionais, cujas características básicas são apresentadas na Tabela 1, o EXPACS e o QARM. Há outros códigos computacionais e ferramentas, mas que não serão abordados neste trabalho. 
Tabela 1: Características básicas dos códigos de cálculo utilizados no trabalho. Extraído de (FEDERICO, 2011).

\begin{tabular}{lcccc}
\hline Código & Disponibilidade & $\begin{array}{c}\text { Grandeza de } \\
\text { resposta }\end{array}$ & $\begin{array}{c}\text { Fatores de conversão } \\
\text { para dose }\end{array}$ & Tipo de cálculo \\
\hline EXPACS & Pacote & $\begin{array}{c}E, \dot{E}, H^{*}(10), \\
\dot{H}^{*}(10)\end{array}$ & $\begin{array}{c}\text { ICRP 60 ou } \\
\text { ICRP 103 }\end{array}$ & Coordenada fixa \\
\hline QARM & On-line & $\begin{array}{c}E, \dot{E}, H^{*}(10), \\
\dot{H}^{*}(10)\end{array}$ & ICRP 74 & $\begin{array}{c}\text { Rota, coordenada } \\
\text { fixa }\end{array}$ \\
\hline \multicolumn{2}{c}{$\begin{array}{l}E=\text { dose efetiva; } \dot{E}=\text { taxa de dose efetiva; } H^{*}(10)=\text { equivalente de dose ambiente; } \dot{H}^{*}(10)=\text { taxa de } \\
\text { equivalente de dose ambiente }\end{array}$} &
\end{tabular}

O EXPACS (Excel-based Program for calculating Atmospheric Cosmic-ray) (SATO, 2006; SATO, 2008) não é considerado propriamente um código de cálculo de dose, mas trata-se de ferramenta e consiste em uma planilha que permite a determinação da taxa de dose em qualquer posição geográfica e altitude desejadas. Foi desenvolvido a partir de simulações da interação da radiação cósmica na atmosfera, pelo método de Monte Carlo empregando o código computacional PHITS. Os resultados destas simulações foram parametrizados em um código analítico denominado PARMA.

Para a utilização do EXPACS, o usuário insere como parâmetros de entrada a altitude e as coordenadas geográficas do ponto desejado, ou ainda a rigidez de corte vertical. Os parâmetros geofísicos são recuperados pelo código de uma biblioteca interna, sendo necessário fornecer a data desejada, ou o potencial de desaceleração solar na data desejada ou, ainda, a contagem de nêutrons obtida na estação de nêutrons de Thule (Groenlândia).

Os resultados podem ser obtidos na forma de taxa de dose efetiva ou taxa de equivalente de dose ambiente. Os resultados são apresentados separadamente por componente da radiação (nêutrons, prótons, núcleos de hélio, múons positivos e negativos, elétrons, pósitrons e fótons).

O QARM (Quotid Atmospheric Radiation Model) (LEI, 2004) é um código de cálculo de dose que utiliza uma base de dados gerados por cálculos de propagação da radiação cósmica incidente no topo da atmosfera realizados pelo método de Monte Carlo. Este código é acessível por meio de uma plataforma web e permite calcular taxas de dose para posições fixas, doses acumuladas em rotas definidas pelo usuário (dose efetiva ou equivalente de dose ambiente). Para a utilização 
do QARM, o usuário insere como parâmetros de entrada as coordenadas geográficas ou os aeroportos de origem e destino, o índice Kp (índice de perturbação geomagnético planetário) ou a contagem de nêutrons de alguns observatórios.

\section{MATERIAIS E MÉTODOS}

Para a realização deste trabalho foram realizadas várias simulações com códigos EXPACS e QARM, no intervalo de altitude de 0 a 20 km, variando os parâmetros de rigidez de corte vertical e de potencial de desaceleração solar.

Os parâmetros utilizados para os cálculos por meio dos códigos estão listados na Tabela 2.

Tabela 2: Parâmetros utilizados nos códigos para a obtenção dos fluxos e valores de dose no trabalho

\begin{tabular}{|c|c|c|c|}
\hline Código & Altitude [km] & $\begin{array}{c}\text { Rigidez de corte } \\
\text { vertical [GV] }\end{array}$ & $\begin{array}{c}\text { Potencial de } \\
\text { desaceleração solar [MV] }\end{array}$ \\
\hline EXPACS & $\begin{array}{l}0 \text { a } 20 \mathrm{~km} \text {, com intervalo de } 1 \\
\mathrm{~km} .\end{array}$ & $\begin{array}{c}0 \text { (polar) }^{1} \\
17 \text { (equatorial })^{1}\end{array}$ & $\begin{array}{c}465(\text { mínimo })^{1} \\
1700(\text { máximo })^{1}\end{array}$ \\
\hline QARM & $\begin{array}{c}1 \mathrm{~m} \text {, de } 1 \text { a } 19 \mathrm{~km} \text {, com } \\
\text { intervalo de } 2 \mathrm{~km} \mathrm{e}, 20 \mathrm{~km} \text {. }\end{array}$ & $\begin{array}{c}0 \text { (polar }^{2} \\
17 \text { (equatorial }^{3}\end{array}$ & $\begin{array}{l}465{\text { (mínimo })^{4}}^{1715(\text { máximo })^{5}}\end{array}$ \\
\hline
\end{tabular}

A partir dos fluxos das partículas obtidos por meio dos códigos EXPACS e QARM, foram determinados os equivalentes de dose ambiente para cada uma das partículas e normalizadas pelo equivalente de dose ambiente total (considerando-se todas as partículas). Desta forma pode-se determinar a contribuição de cada partícula no equivalente de dose ambiente total em função dos parâmetros anteriormente citados.

A partir dos dados do EXPACS, foram calculadas as energias efetivas ponderadas em função do equivalente de dose ambiente para as partículas que mais contribuem no equivalente de dose ambiente total.

A energia efetiva das partículas ponderadas em função do equivalente de dose ambiente, 
$E_{\theta f\left(H^{*}(10)\right)}$, pode ser calculada por meio da equação (1):

$$
\varepsilon_{e f\left(H^{*}(10)\right)}=\frac{\int_{0}^{\infty} \varepsilon H^{*}(10)(\varepsilon) d \varepsilon}{\int_{0}^{\infty} H^{*}(10)(\varepsilon) d \varepsilon}
$$

onde $\varepsilon$ é a energia da partícula e $\mathrm{H}^{*}(10)(\varepsilon)$ é o equivalente de dose ambiente da partícula na energia $\varepsilon$.

\section{RESULTADOS E DISCUSSÕES}

As contribuições das principais partículas no equivalente de dose ambiente em função da altitude, nas diferentes condições de potencial solar e de rigidez de corte vertical, calculadas por meio dos códigos EXPACS e QARM são apresentadas nas Figuras 1 a 4.

Figura 1: Contribuição relativa de cada partícula no equivalente de dose ambiente $H^{*}(10)$ total em função da altitude nas condições de mínimo solar numa região polar, determinadas por meio dos códigos EXPACS e QARM.

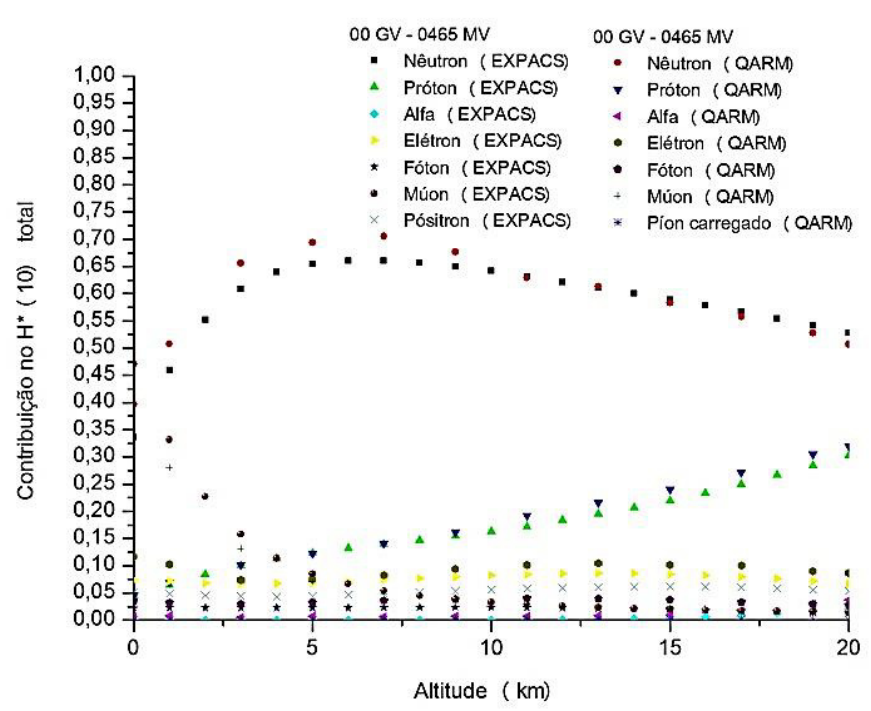


Figura 2: Contribuição de cada partícula no equivalente de dose ambiente $\mathrm{H}^{*}(10)$ total em função da altitude nas condições de máximo solar numa região polar, determinadas por meio dos códigos EXPACS e QARM.

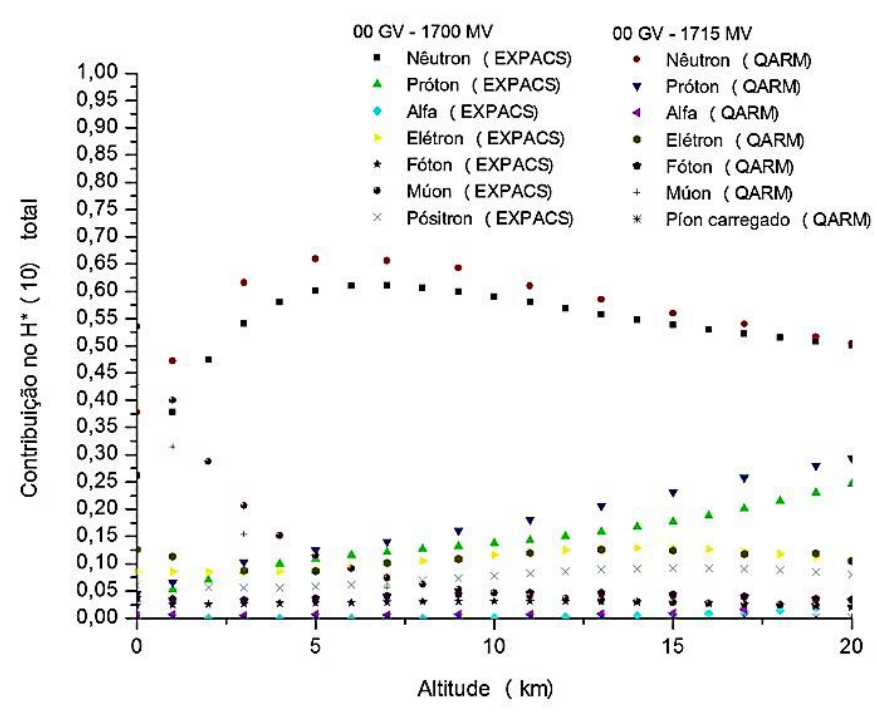

Figura 3: Contribuição de cada partícula no equivalente de dose ambiente $\mathrm{H}^{*}(10)$ total em função da altitude nas condições de mínimo solar numa região equatorial, determinadas por meio dos códigos EXPACS e QARM.

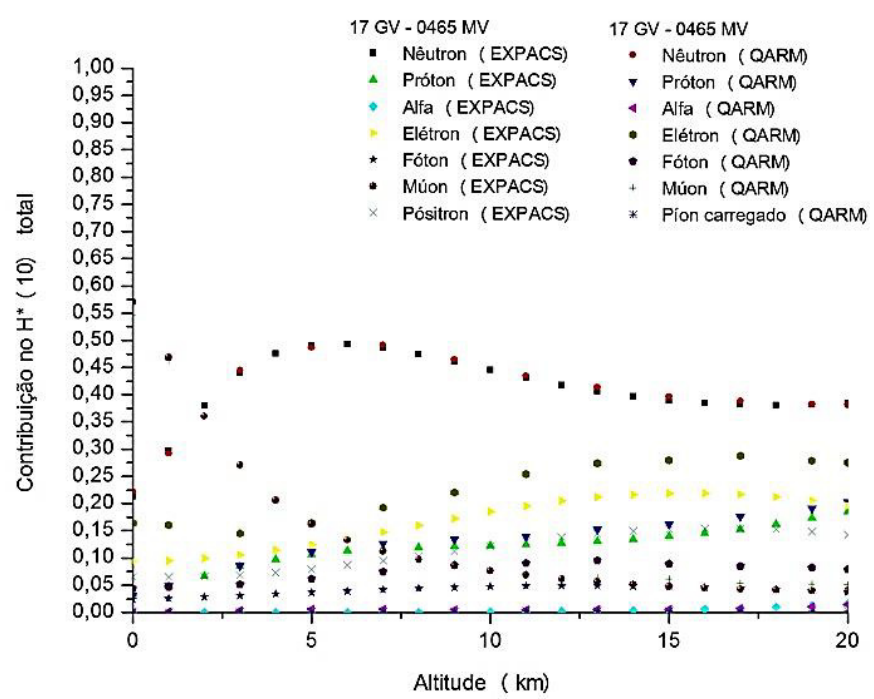

Figura 4: Contribuição de cada partícula no equivalente de dose ambiente $H^{*}(10)$ total em fun- 
ção da altitude nas condições de máximo solar numa região equatorial, determinadas por meio dos códigos EXPACS e QARM

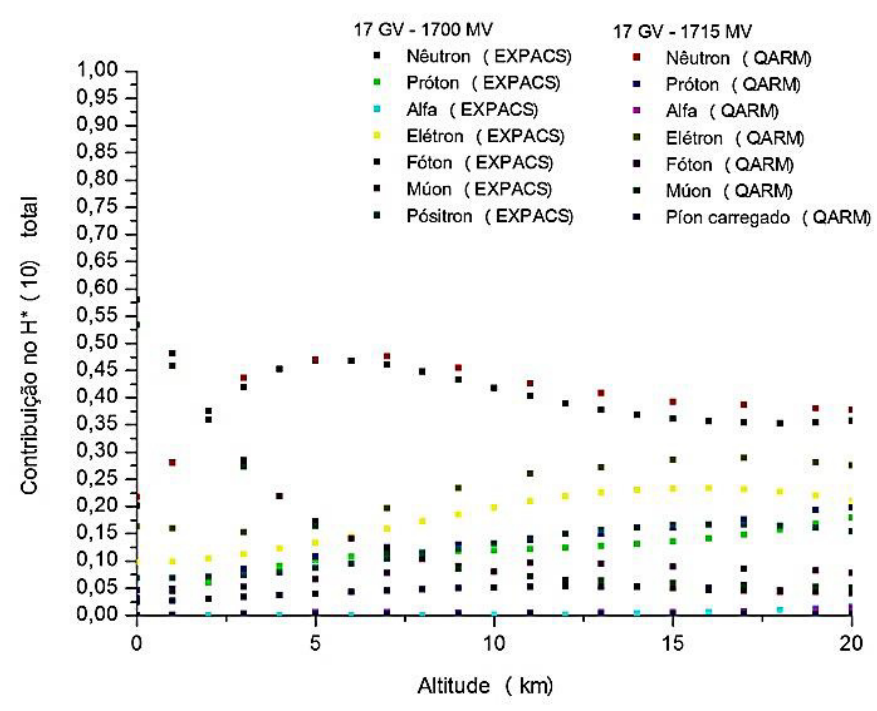

Os resultados apresentados mostram que, acima de $3 \mathrm{~km}$, os nêutrons contribuem no equivalente de dose ambiente total com a maior fração, e sua contribuição máxima ocorre em um intervalo entre 5 e $7 \mathrm{~km}$ de altitude, após o qual sua contribuição no equivalente de dose ambiente total começa a diminuir com o aumento da altitude. Por outro lado, as contribuições devidas a prótons e alfas aumentam em função da altitude enquanto a contribuição devida aos múons diminui.

De forma semelhante aos nêutrons, as contribuições devidas a elétrons e pósitrons possuem um máximo na região em torno de $16 \mathrm{~km}$ enquanto que para fótons possui um máximo na região de $12 \mathrm{~km}$.

As contribuições das partículas no equivalente de dose ambiente além de variarem em função da altitude também variam em função da rigidez de corte vertical e em função do potencial de desaceleração solar.

Com o aumento da rigidez de corte vertical, as contribuições de nêutrons e prótons diminuem, enquanto que as contribuições de alfas, múons, elétrons, pósitrons e fótons aumentam. As contribuições variam do mesmo modo com o aumento do potencial de desaceleração solar.

Outra observação é que com o aumento da rigidez de corte vertical, a contribuição de elétrons se torna mais significativa do que a contribuição dos prótons, chegando a aumentar quase duas 
vezes ao variar-se a rigidez de corte vertical de $0 \mathrm{GV}$ à $17 \mathrm{GV}$.

Um resumo desta análise é apresentado na Tabela 3.

Tabela 3: Resumo do comportamento das principais partículas no equivalente de dose ambiente total e suas variações em função dos parâmetros avaliados neste trabalho.

\begin{tabular}{lccc}
\hline \multicolumn{1}{c}{ Partícula } & Altitude & $\begin{array}{c}\text { Rigidez de corte } \\
\text { vertical }\end{array}$ & $\begin{array}{c}\text { Potencial de desaceleração } \\
\text { solar }\end{array}$ \\
\hline Nêutrons & Máximo entre 5 e $7 \mathrm{~km}$ & Diminui & Diminui \\
\hline Prótons & Aumenta & Diminui & Diminui \\
\hline Alfas & Aumenta & Diminui & Praticamente não varia \\
\hline Múons & Diminui & Aumenta & Aumenta \\
\hline Elétrons & $\begin{array}{c}\text { Máximo em torno de } \\
12 \mathrm{~km}\end{array}$ & Aumenta & Aumenta \\
\hline Pósitrons & $\begin{array}{c}\text { Máximo em torno de } \\
16 \mathrm{~km}\end{array}$ & Aumenta & Aumenta \\
\hline Fótons & Máximo em torno de \\
$12 \mathrm{~km}$ & Aumenta & Aumenta \\
\hline $\begin{array}{l}\text { Píons } \\
\text { carregados }\end{array}$ & Aumenta & Aumenta & Aumenta \\
\hline
\end{tabular}

Foram calculados os espectros em energia das principais partículas contribuintes no equivalente de dose ambiente total em função da altitude, da rigidez de corte vertical e do potencial de desaceleração solar. Os resultados são apresentados nas Figuras 5 a 9.

Figura 5: Espectro em energia da taxa de equivalente de dose ambiente para nêutrons em função da altitude
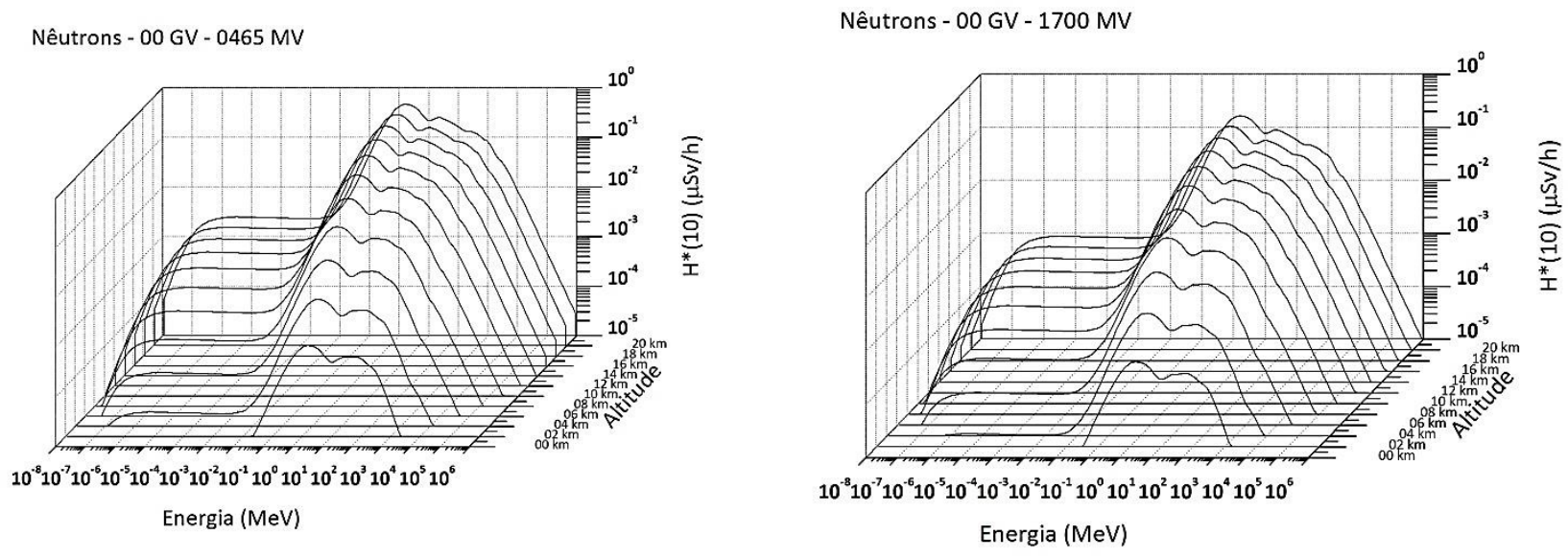

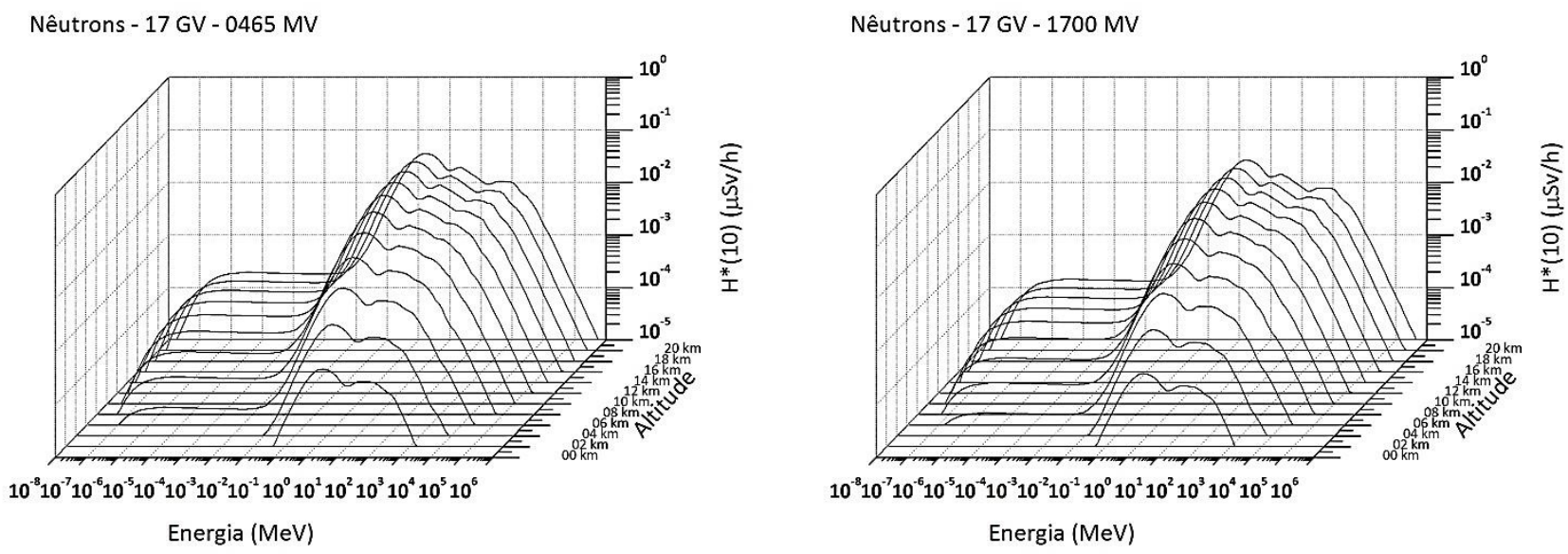

O espectro de nêutrons não sofre grandes alterações na sua forma, porém podemos observar que a maior variação ocorre com a variação da rigidez de corte vertical, havendo uma diminuição da taxa de equivalente de dose ambiente na energia máxima do espectro em até 13 vezes quando há um aumento na rigidez de corte vertical, de uma região polar $(00 \mathrm{GV})$ para uma região equatorial (17 GV). Por outro lado, o aumento da atividade solar não influencia significativamente na taxa de equivalente de dose ambiente na energia máxima do espectro, diminuindo no máximo cerca de 3 vezes, pois a produção de nêutrons ocorre na atmosfera, dependendo principalmente dos processo de interação da RC primária com as partículas da atmosfera.

Figura 6: Espectro em energia da taxa de equivalente de dose ambiente para prótons em função da altitude
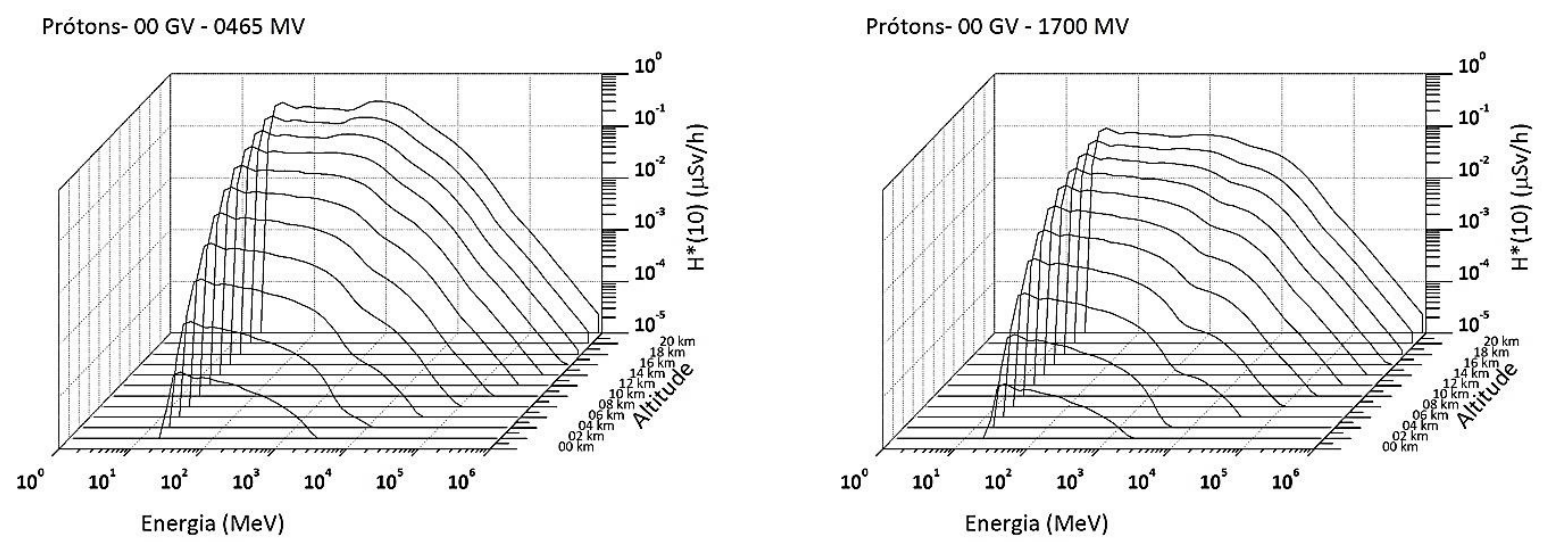

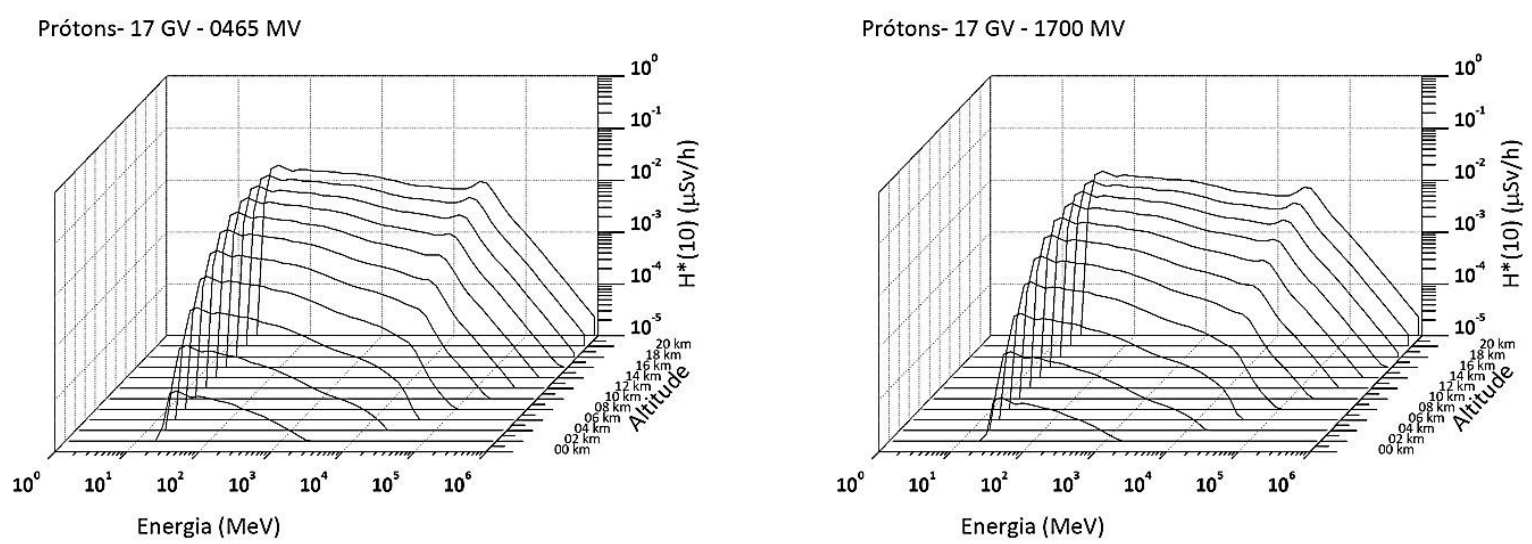

O espectro de prótons sofre significativas alterações na sua forma, podemos observar que a maior variação ocorre com a variação da rigidez de corte vertical, havendo uma diminuição da taxa de equivalente de dose ambiente na energia máxima do espectro em até 16 vezes quando há um aumento na rigidez de corte vertical, de uma região polar $(00 \mathrm{GV})$ para uma região equatorial (17 GV). Por outro lado, o aumento da atividade solar não influencia significativamente na taxa de equivalente de dose ambiente na energia máxima do espectro, diminuindo no máximo cerca de 3 vezes, pois os prótons são a principal componente da RC primária que penetra e interagem com a atmosfera, produzindo a RC secundária.

No caso dos prótons, pode ser observado claramente um achatamento do espectro com o aumento da rigidez de corte vertical. Tal comportamento é resultado da deflexão magnética das partículas que possuem rigidez magnética menor do que a rigidez de corte vertical do campo, naquela localização. Da Figura 6 pode-se verificar que tal deflexão não é observada para prótons com energias superiores à, aproximadamente, $2 \times 10^{4} \mathrm{MeV}$, para os quais o campo magnético terrestre não constitui obstáculo significativo à penetração da partícula. 
Figura 7: Espectro em energia da taxa de equivalente de dose ambiente para elétrons em função da altitude
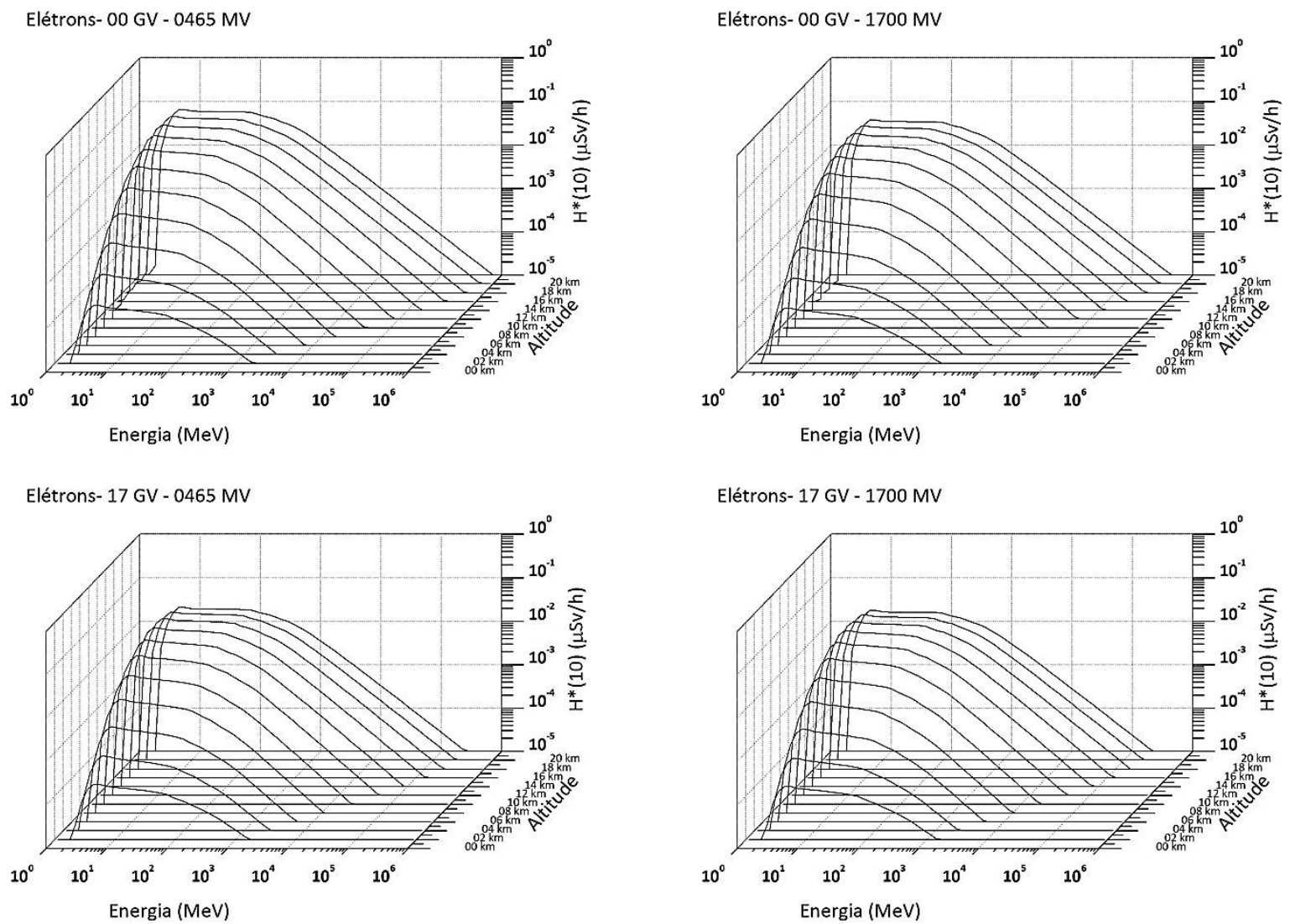

Figura 8: Espectro em energia da taxa de equivalente de dose ambiente para pósitrons em função da altitude
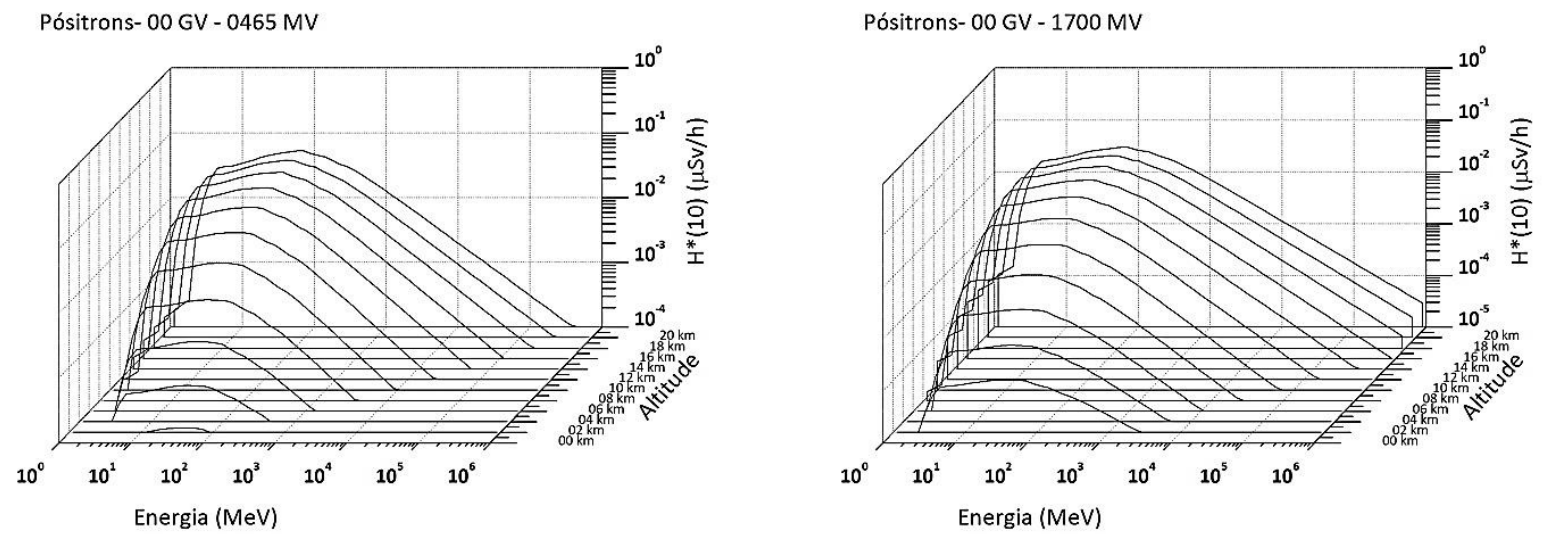

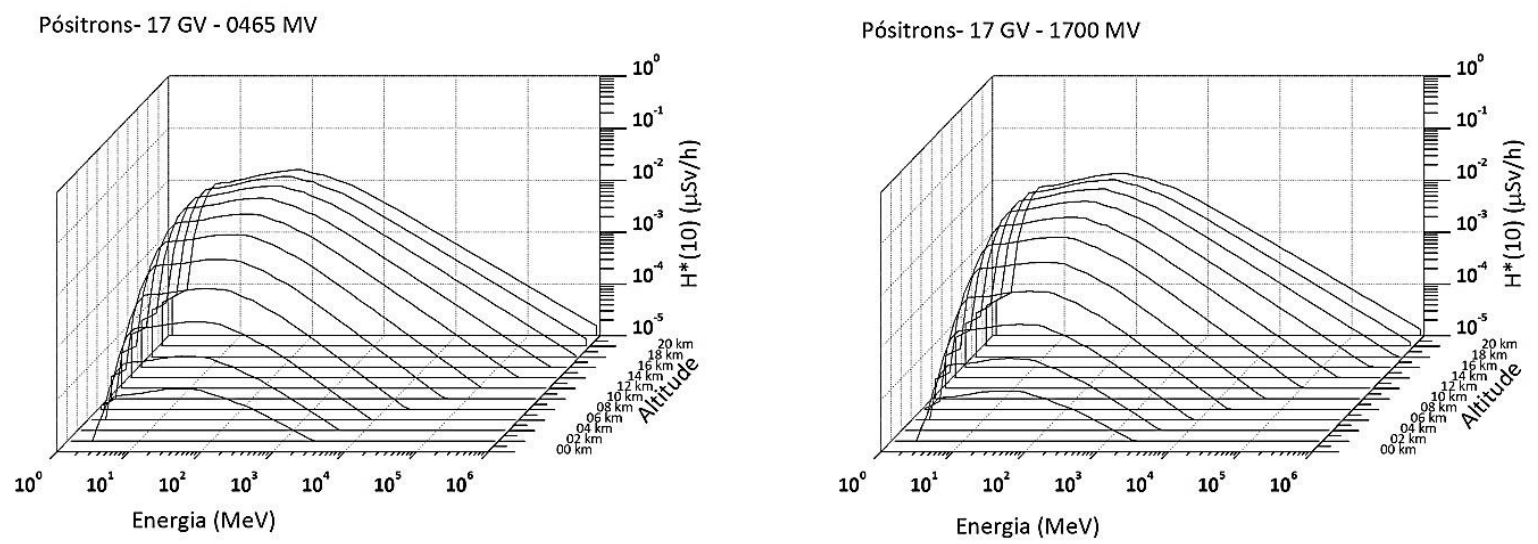

No caso da Figura 7 e da Figura 8 (elétrons e pósitrons), o comportamento é bastante similar, uma vez que os espectros não sofrem grandes alterações na sua forma. Porém podemos observar que a maior variação ocorre com a variação da rigidez de corte vertical, havendo uma diminuição da taxa de equivalente de dose ambiente na energia máxima do espectro em até 3 vezes quando há um aumento na rigidez de corte vertical, de uma região polar (00 GV) para uma região equatorial (17 GV). Por outro lado, o aumento da atividade solar não influencia significativamente na taxa de equivalente de dose ambiente na energia máxima do espectro, diminuindo no máximo cerca de 2 vezes .

Esse comportamento para os elétrons e pósitrons pode ser explicado por dois fatores: primeiro pelo fato de que tais partículas possuem baixa rigidez magnética, devido à seu menor momento em comparação com os prótons, de forma que as alterações ocorrem no espectro como um todo, não resultando em alterações significativas na forma do espectro. Em segundo lugar é necessário considerar que tais partículas são produzidas em quantidade durante os processos de interação de outras partículas, tais como nêutrons, prótons e fótons nas moléculas da atmosfera, em várias altitudes, de forma que sua produção contínua torna pouco evidente o efeito da rigidez de corte do campo. 
Figura 9: Espectro em energia da taxa de equivalente de dose ambiente para fótons em função da altitude
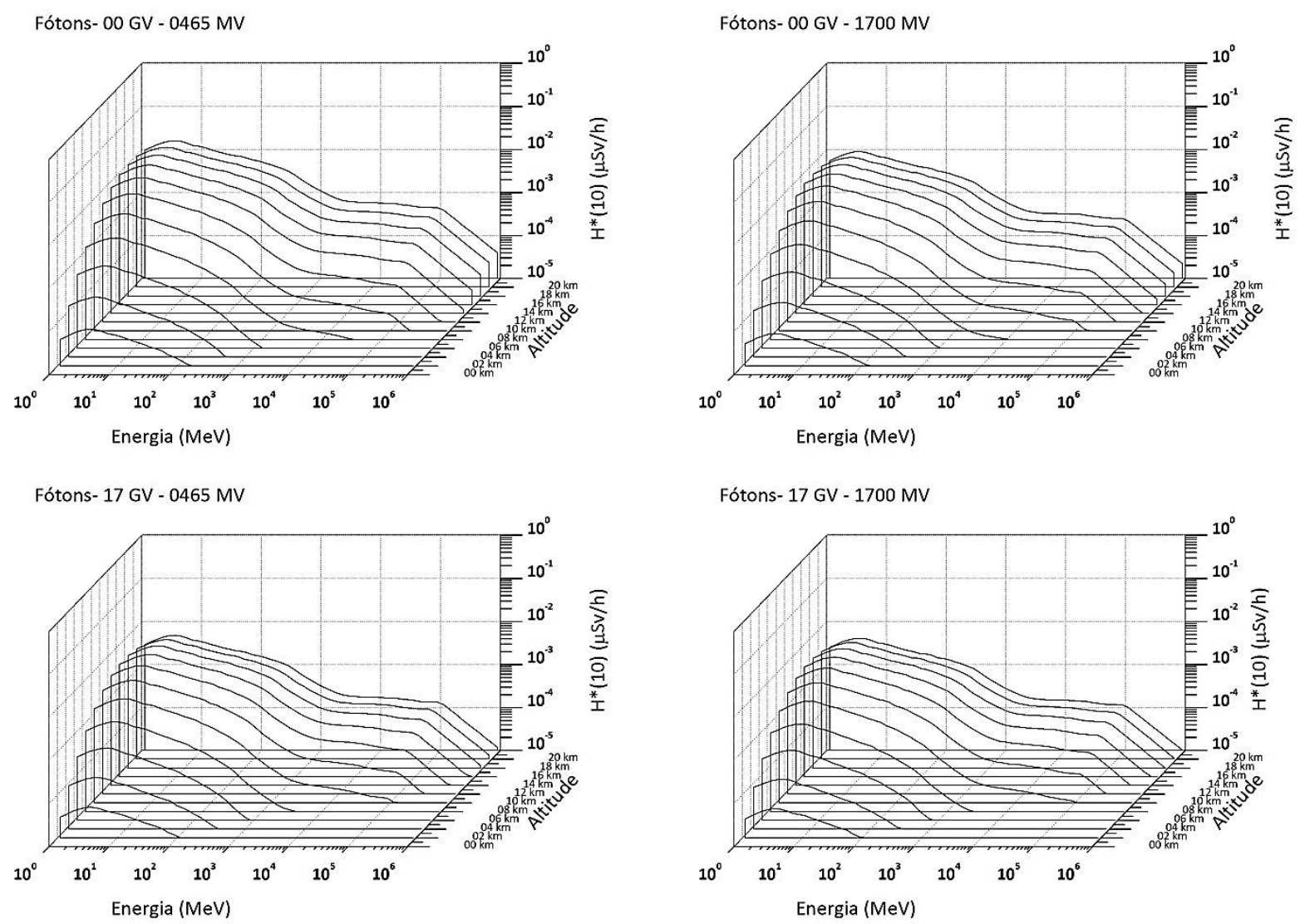

O espectro de fótons não sofre grandes alterações na sua forma, porém podemos observar que a maior variação ocorre com a variação da rigidez de corte vertical, havendo uma diminuição da taxa de equivalente de dose ambiente na energia máxima do espectro em até 3 vezes quando há um aumento na rigidez de corte vertical, de uma região polar $(00 \mathrm{GV})$ para uma região equatorial (17 GV). Por outro lado, o aumento da atividade solar não influência significativamente na taxa de equivalente de dose ambiente na energia máxima do espectro, diminuindo no máximo cerca de 2 vezes. É importante observar que fótons não sofrem influência direta do campo magnético terrestre, mas são produzidos em grande quantidade nos processos de interação de outras partículas com as moléculas da atmosfera, de forma que sua variação com o parâmetro rigidez de corte vertical é resultante principalmente de variações nas outras partículas que compõem a radiação cósmica na atmosfera e que são precursores de fótons, tais como prótons, nêutrons, 
elétrons, pósitrons e etc.

Conforme apresentado na Tabela 4, as partículas que mais contribuem para o equivalente de dose ambiente total em altitudes de interesse de voo, ou seja, dentro do espaço aéreo RVSM (Reduced Vertical Separation Minimum ou Mínimo de Separação Vertical Reduzido), de 29000 e 41000 pés (aproximadamente entre 8800 e 12500 m) são, em ordem de contribuição, nêutrons, prótons, elétrons e pósitrons, representando acima de $80 \%$ da dose total.

Tabela 4: Contribuição média no equivalente de dose ambiente total [\%] dentro da RSVM.

\begin{tabular}{ccccccccc}
\hline \multirow{2}{*}{$\begin{array}{c}\text { Rigidez de } \\
\text { corte vertical }\end{array}$} & $\begin{array}{c}\text { Potencial de } \\
\text { desaceleração } \\
\text { solar }\end{array}$ & \multicolumn{6}{c}{ Contribuição no equivalente de dose ambiente total [\%] } \\
\cline { 5 - 9 }$[\mathrm{GV}]$ & Nêutron & Próton & Alfa & Múon & Elétron & Pósitron & Fóton \\
\hline 0 & 465 & 63,54 & 16,93 & 0,10 & 3,21 & 8,29 & 5,63 & 2,30 \\
\hline 0 & 1700 & 58,36 & 14,14 & 0,15 & 4,53 & 11,77 & 7,95 & 3,10 \\
\hline 17 & 465 & 43,95 & 12,46 & 0,12 & 7,43 & 18,82 & 12,50 & 4,72 \\
\hline 17 & 1700 & 41,12 & 12,09 & 0,13 & 7,81 & 20,15 & 13,58 & 5,12 \\
\hline
\end{tabular}

As energias efetivas, em relação ao equivalente de dose ambiente, dos nêutrons, prótons, elétrons, pósitrons e fótons, em função da altitude são apresentadas na Figura 10.

Figura 10: Energias efetivas em dos nêutrons, prótons, elétrons, pósitrons e fótons em função da altitude.
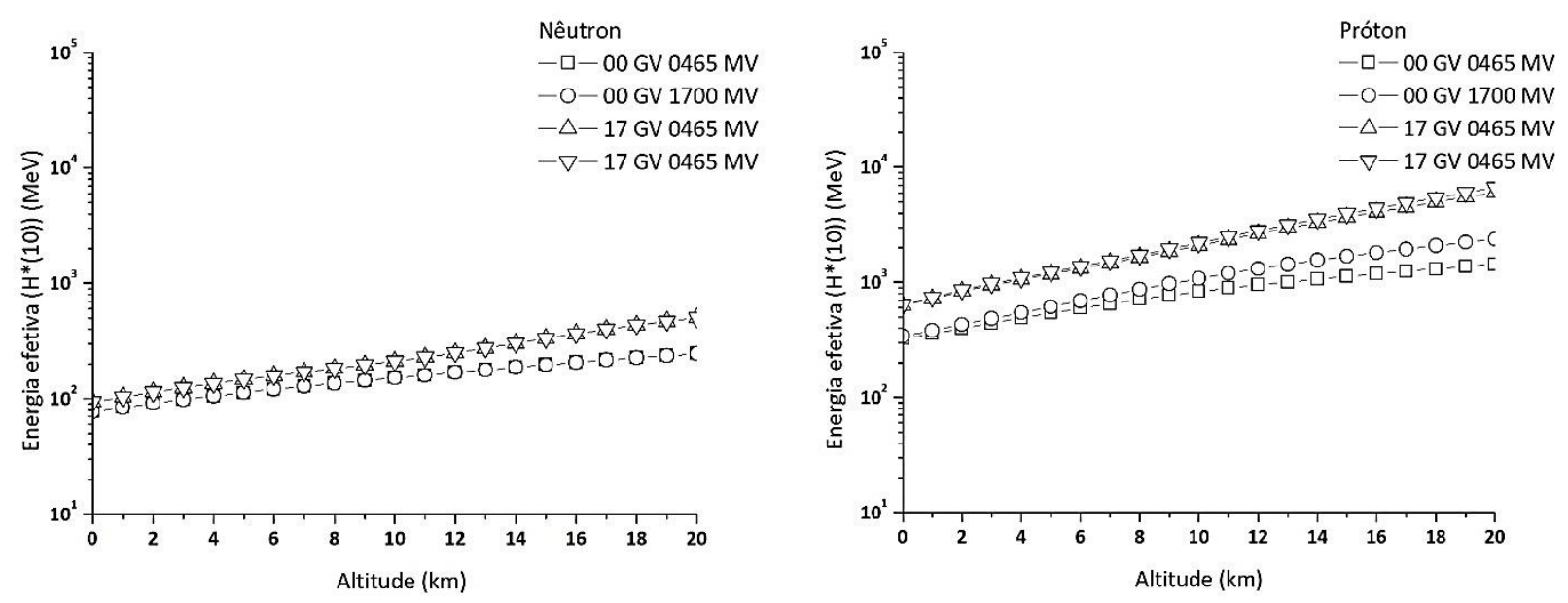

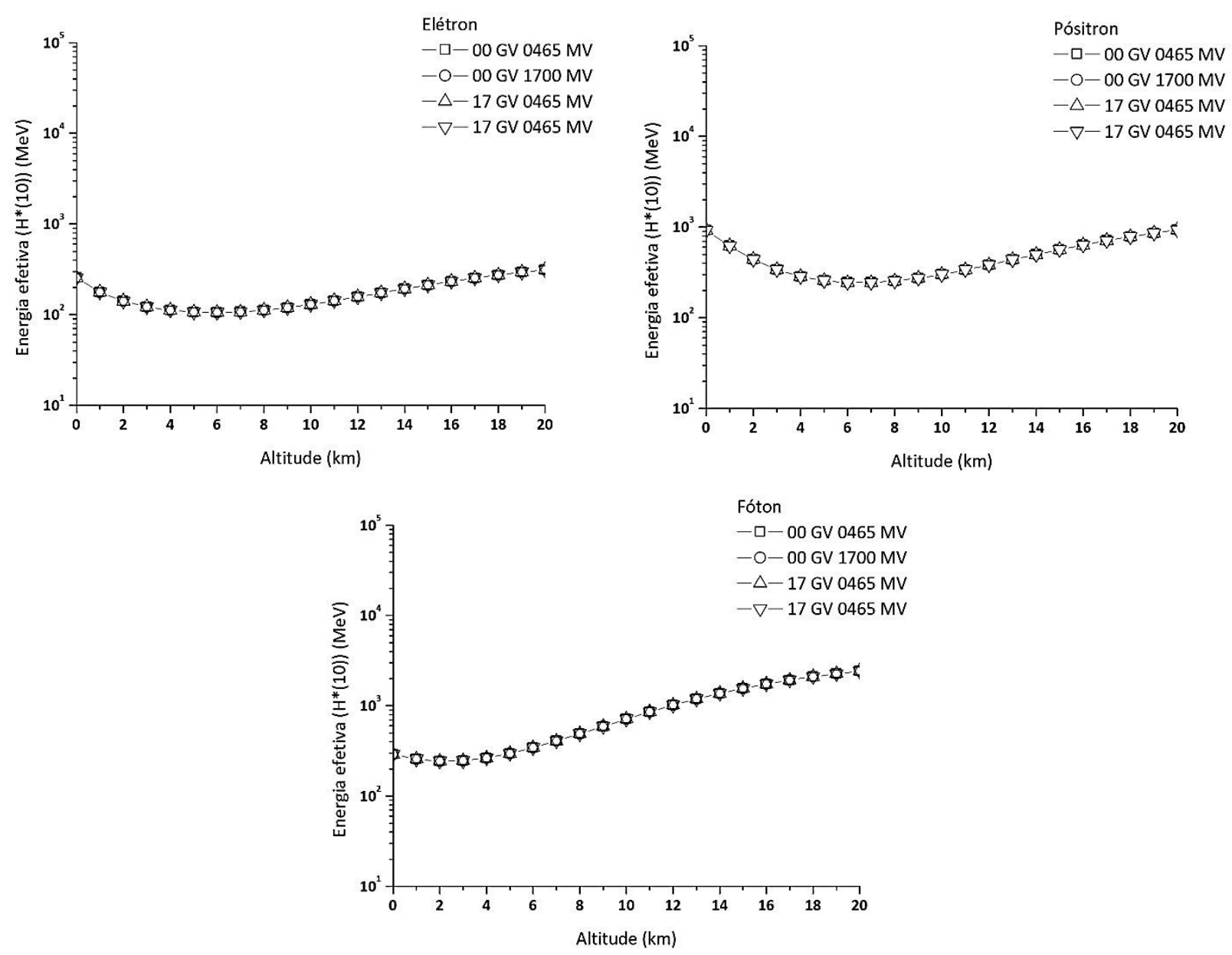

Da Figura 10 observa-se que as únicas partículas que sofrem variação na sua energia média em função dos parâmetros geomagnéticos e solares são os nêutrons e os prótons.

A energia efetiva dos nêutrons aumenta com o aumento da rigidez de corte vertical, e é independente do parâmetro potencial de desaceleração solar. Já para prótons, a energia efetiva aumenta com o aumento da rigidez de corte vertical e também aumenta ligeiramente com o potencial de desaceleração solar, sendo este último aumento mais significativo para rigidez de corte vertical mais baixa.

\section{CONCLUSÕES}

Observa-se que os nêutrons são as partículas preponderantes na composição do equivalente de 
dose ambiente, seguidos dos prótons, para regiões do globo com rigidez de corte vertical baixa ou dos elétrons e pósitrons, no caso de regiões do globo com rigidez de corte vertical alta. As faixas de energia mais importantes para o equivalente de dose ambiente em altitudes de interesse para aviação são de $10^{-8}$ a $10^{6} \mathrm{MeV}$ para nêutrons, de 10 a $10^{6} \mathrm{MeV}$ para prótons e de 1 a $10^{6}$ $\mathrm{MeV}$ para elétrons e pósitrons. As demais partículas contribuem de forma menos significativa (menos de 15\%) para o equivalente de dose ambiente total.

Como apresentado na introdução deste trabalho, este estudo busca estabelecer a base fundamental para a descrição da origem primária da dose recebida por tripulações, quais as partículas que causam as principais contribuições, como esta contribuição varia em função da altitude, rigidez de corte vertical e potencial de desaceleração solar e as energias das principais partículas contribuintes, estabelecendo assim os fundamentos para o projeto de um sistema dosimétrico apropriado para este tipo de campo misto de radiação ionizante no interior de aeronaves e em solo.

\section{AGRADECIMENTO}

À CAPES (Programa Pró-estratégia) pela bolsa de mestrado do primeiro autor, ao CNPq (processos 402209/2013-3 e 481432/2013-2) e FINEP (projeto CITAR) pelo apoio parcial e ao ITA e IEAv pelo suporte e oportunidade de estudar e agregar conhecimento. 


\section{REFERÊNCIAS}

BARTLETT, D. T. Radiation protection aspects of the cosmic radiation exposure of aircraft crew. Radiation Protection Dosimetry, v. 109, n. 4. p. 349-355, 2004.

DURANTE M. e CUCINOTTA, F. Physical basis of radiation protection in space travel. Reviews of Moderns Physics, v 83, p. 1245-1281, 2011.

FEDERICO C. A. et al. Cosmic Radiation effects on aircrew and avionics in the brazilian airspace. Em: Simpósio Aeroespacial Brasileiro - SAB 2012, 2012, São José dos Campos, São Paulo.

FEDERICO, C. A. Dosimetria da radiação cósmica no interior de aeronaves no espaço aéreo brasileiro. 2011. 172f. Tese (Doutorado em Ciências na Área de Tecnologia Nuclear) - Instituto de Pesquisas Energéticas e Nucleares, São Paulo.

FEDERICO, C. A. et al. Effects of cosmic radiation in aircrafts: A discussion about aircrew over South America. Journal of Aerospace Technology and Management, v. 4, n. 2. p. 219-255, 2012.

FEDERICO, C. A. et al. Neutron spectra measurements in the south Atlantic anomaly region. Radiation Measurements, v. 45, n. 10. p. 1526-1528, 2010.

HAJEK, M. et al. A TLD-based personal dosemeter system for aircrew monitoring. Radiation Protection Dosimetry, v. 110, n. 1-4, p. 337-341, 2004.

ICRP - International Commission on Radiological Protection. 1990 Recommendations of the International Commission on Radiological Protection. ICRP Publication 60. Ann. ICRP 21 (1-3), 1991.

ICRP - International Commission on Radiological Protection. Conversion coefficients for use in radiological protection against external radiation. ICRP Publication 74. Ann. ICRP 26 (34), 1996.

ICRP - International Commission on Radiological Protection. The 2007 Recommendations of the International Commission on Radiological Protection. ICRP Publication 103. Ann. ICRP 37 (2-4), 2007. 
LEI, F. et al. An Atmospheric Radiation Model Based on Response Matrices Generated by Detailed Monte Carlo Simulations of Cosmic Ray Interactions. IEEE Transactions on Nuclear Science, v. 51, n. 6, p. 3442-3451, 2004.

LIM, M. K. Cosmic Ray: are Air Crew at Risk, Occupational \& Environmental Medicine, v. 59, p. 428-433, 2002.

SATO T. e NIITA, K. Analytical Functions to Predict Cosmic-Ray Neutron Spectra in the Atmosphere, Radiation Research, v. 166, p. 544-555, 2006.

SATO T. et al. Development of PARMA: PHITS'-based Analytical Radiation Model in the Atmosphere. Radiation Research, v. 170, p. 244-259. 2008. 